\title{
Research on Mass Imbalance Fault of Wind Turbine Based on Virtual Prototype
}

\author{
Ping Zhao , Xiaoguang Li and Liu Yang \\ CRRC Zhuzhou Electric Locomotive Research Institute, Zhuzhou, 412001
}

\begin{abstract}
Wind turbine virtual prototype was set up with SIMPACK software. The influence of different mass in different blades was researched by changing size and position of imbalance mass. Results showed that the period of imbalanced moment inducted by different size of imbalance mass imposing blades at different position was the same as the wind turbine rotor, but the amplitude and phase was different. Electricity generation during starting was associated with not only the size of mass but also the position. After starting, wind turbine would keep the stable state in simulation, power and revolution speed and pitch angle curves would wave periodically, the periods were all the same as the wind turbine rotor, the size of imbalance masses were bigger, the fluctuation amplitude of curves were greater.
\end{abstract}

\section{Introduction}

In recent years, wind energy, as a new, safe and reliable, clean energy, has been given more and more attentions and large-scale developed by international which possess abundant resources[1-2]. Developing wind electricity has also been an important measure to carry out the sustainable energy development strategy of China. As the capacity of single wind turbine has become larger and larger, the impeller diameter has become longer and longer, the flexibility of wind turbine has become stronger, and influence of impeller imbalanced faults has received more and more attentions, such as the increasing of huge vibration, drive train vibration, and parts fatigue stress of whole wind turbine caused by it. And the sand abrasion, blade icing, loosen of the inner filling material will lead to mass unbalance of impeller.

Many researchers have carried out relevant researches aim at impeller imbalance faults of wind turbine generator set. German research institution ISET found that [3] it could be gotten the mass imbalance faults of wind turbine through testing electrical signal of generator, however, they didn't offer any theoretical explanation of this diagnostic technique. RONNY and the others [4-5] from university of linz in Austria analyzed vibration characteristics wind turbine. Identify the size and direction of impeller imbalance mass through using reverse engineering research methods. JIANG [6-7] carried out experiments aimed at three malfunction of impeller including mass imbalance, asymmetric aerodynamic force, yaw misalignment, using way which is based on vibration spectrum analysis to research on main shaft vibration of impeller caused by mass imbalance. Yang Tao [8-9] conducted simulation for impeller imbalance faults including mass imbalance, asymmetric aerodynamic force malfunction, they pointed out under the state of mass imbalance faults, wave of electric power was resulted from the wave of impeller output torque caused by gravity of imbalance mass, and under the state of asymmetric aerodynamic force faults, because of the vibration of tower leads to the wave of aerodynamic force on impeller, then leads to wave of torque, and at last leads to the wave of electric power. In other words, aerodynamic force model and tower vibration model are coupled. Guo Yuanchao [10] compared the changing situation of equivalent fatigue loading at all important point of load of wind turbine generator in different condition of maximum mass eccentricity, and it explained that mass imbalance of wind wheel makes serious influence on fatigue loads.

The research methods above are mainly based on two class methods, one of it is a fault diagnosis method based on vibration, and this method need to install a large number of sensors, low reliability, but high costing. Another one is based on simulation analytical calculation, modeling and simulation of faults can make a more profound understanding of malfunction formulation mechanism and influences, and it will be easier to proceed calculation and comparison for different malfunction signal, however, this kind of simulation is based on physical parameters used of wind turbine without considering dynamical behavior of the wind turbine, and neither do consider the relevant connected relations and coupling of impeller, transmission chain and tower-silo, so that this simulation can only aim at one physical quantity of electric power.

This paper infers the influence that the size of impeller imbalance mass moment of wind turbine do on 
electric power in theory, and wind turbine real simulation was set up with many-body dynamics software of SIMPACK, makes simulation on location distribution on wind turbine of imbalance mass and mass of different size to load on the same direction of same impeller to research on the influences it makes on generator power, rotate speed and blade variable pitch respectively.

\section{Wind turbine mass imbalance moment}

Wind turbine generator of three-vanned is showed in Figure 1, the impeller is rotates clockwise at speed of $\omega$, suppose the initial position of blade 1 is right above, when impeller turn a angle of $w t$ at speed of $\omega$, the mass moment of impeller $T_{g}$ is:

$$
T_{g}=G_{1} r_{1} \sin (w t)+G_{2} r_{2} \sin \left(w t+\frac{2 \pi}{3}\right)+G_{3} r_{3} \sin \left(w t-\frac{2 \pi}{3}\right)
$$

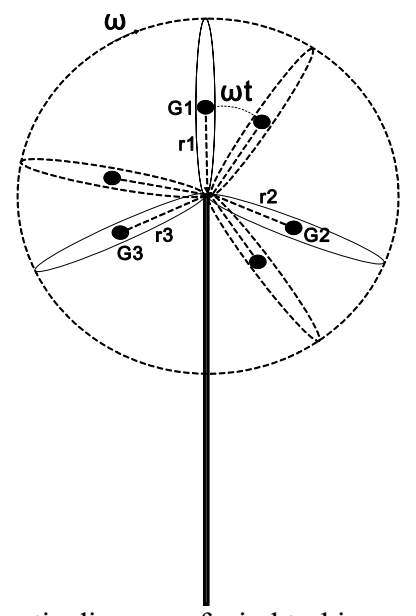

Figure 1. Schematic diagram of wind turbine mass distribution.

Unfold the formula (1), it can be inferred as follows:

$T_{g}=\left(G_{1} r_{1}-\frac{1}{2} G_{2} r_{2}-\frac{1}{2} G_{3} r_{3}\right) \sin (w t)+\frac{\sqrt{3}}{2}\left(G_{2} r_{2}-G_{3} r_{3}\right) \cos (w t)$

Let

$$
k_{1}=G_{1} r_{1}-\frac{1}{2} G_{2} r_{2}-\frac{1}{2} G_{3} r_{3}, k_{2}=\frac{\sqrt{3}}{2}\left(G_{2} r_{2}-G_{3} r_{3}\right),
$$

So that formula (2) can be turned into formula (3).

$$
T_{g}=k_{1} \sin (w t)+k_{2} \cos (w t)
$$

Suppose

$$
\sin \varphi=\frac{k_{2}}{\sqrt{k_{1}^{2}+k_{2}^{2}}}, \cos \varphi=\frac{k_{1}}{\sqrt{k_{1}^{2}+k_{2}^{2}}},
$$

It can be inferred from formula (8) as follow:

$$
T_{g}=\sqrt{k_{1}^{2}+k_{2}^{2}} \sin (w t+\varphi)
$$

From the deduction above, it means all the mass moment that is generated from impeller mass imbalance can be expressed by formula (4). Therefore, moment change period from impeller mass imbalance in different conditions is the same with impeller period of rotation, but here are some differences in amplitude and phase position of mass, and we can infer backwards the mass imbalance situation according to size of amplitude and value of phase position. Suppose that the impeller receives Ta aerodynamic moment, we can get the total moment received by impeller and electric power of generator respectively:

$$
\begin{gathered}
T=T_{\mathrm{a}}+T_{\mathrm{g}} \\
P_{\mathrm{e}}=\left(T_{\mathrm{a}}+T_{\mathrm{g}}\right) \omega / k
\end{gathered}
$$

where, $P_{e}$ represents output power of generator, $\omega$ represents geostationary angular spin rate, $k$ represents transmission ratio of gearbox. From formula (6), we can see that $\omega$ and $k$ are constant, we suppose impeller aerodynamic moment in rotation process $T_{a}$ is also a constant value, so, the change of output power is proportional to impeller imbalance mass moment, change period is the same as imbalance mass moment period.

\section{Virtual prototype model}

Based on the analysis above, this paper use software of SIMPACK to set up wind turbine generator virtual prototype model, topological structure is showed in Figure 2. The model includes impeller, hub, spindle, gearbox model and generator model, we can research on the influences of wind turbine generator caused by different sizes of imbalance mass on different blades through changing the size and position of imbalance mass. Force of wind turbine take way of applying wind load, number 241 of aerodynamic element in SIMPACK software is interface of Aerodyn aerodynamic software. It will calculate aerodynamic through associating simulation form of Aerodyn software at run time. We can achieve simulation of pitch control through force element FE243 (Wind Controller Interface). Add virtual hub of zero mass and virtual variable pitch of zero mass respectively between blade and hub, allowing blade rotate in axial, so that SIMPACK software can call DLL file of dynamic depot in control model directly to achieve variable pitch control when calculate it. For achieving variable pitch control, SIMPACK software usually consider rotate speed of engine rotor and electric power as output, and then, put it into control procedure, variable pitch angle, as the output of control procedure, will be delivered back into dynamic model as the input of SIMPACK, so that to achieve joint simulation.

Hub and spindle use the power force of FE13 SpringDamp Rot Meas Inp Cmp) to represent the bolted connection between them. Spindle connect with rack through main bearing, represented by FE41 (SpringDamp Matrix Cmp). Guide the whole gearbox into the whole model as a substructure. Force element FE5 (Spring-Damp Parallel Cmp) represent flexible support of 
which connect gearbox and rack. The connection between coupling, generator, front and back gearbox is permanent connection, release the rotate DOF in axial between individual couplings, torsional rigidity and damping reflect in force element FE13. There are bearings and electromagnetic force between generator rotor and stator, use FE43 (Bushing Cmp) and FE50 (Force Torque Expression Cmp) to represent them respectively. The same as force element FE5 (Spring-Damp Parallel Cmp) to represent flexible support of which connect generator and rack. A virtual rack of zero mass is built in the bottle of the rack to connect with tower-silo, and the whole wind turbine connects with ground through tower-silo, tower-silo simulates with fully flexible form. Model is proceeded meshing and built main node in ANSYS software at first, and then, guide it into SIMPACK model. Damping $d$ calculations of flexible support and coupling are showed here:

Flexible support: $d_{1}=2 D_{1} \sqrt{K_{1} m_{e q} / n}$,

$D_{1}$ represents damping coefficient, $K_{1}$ represents flexible support rigidity, $n$ represents flexible support quantity, meq represents equivalent mass, $m_{e q}=I / r^{2}, r$ represents radius of rotary arm, $I$ represents rotary inertia for direction of rotation.

Rotary inertia calculation of gearbox flexible support is expressed as follows: $I=I_{\text {bades }}+I_{\text {mainshaft }}+I_{\text {hub }}+I_{\text {housing, }}$, $I_{\text {bades }}$ is rotary inertia of whole impeller relative to spindle, $I_{\text {mainshaft }}$ represents rotary inertia of spindle rotate in axial, $I_{h u b}$ represents the moment of inertia of the wheel around the axial rotation, $I_{\text {housing }}$ represents the moment of inertia of the wheel around the axial rotation.

Rotary inertia calculation of generator flexible support is expressed as follows: $I=I_{\text {rotor }}+I_{\text {stator }}, \quad I_{\text {rotor }}$ is rotary inertia of spindle when generator rotor rotates around spindle, $I_{\text {stator }}$ is rotary inertia of spindle when generator stator and frame rotate around spindle.

Damping calculation formula of coupling is: $d_{2}=2 D_{2} \sqrt{K_{2} I_{2}}$

, $\mathrm{D}_{2}$ is damping coefficient, $K_{2}$ is torsional rigidity, calculation of rotary inertia I are here below:

$$
I=I_{\text {coupling2 }}+I_{\text {coupling } 3}+I_{\text {coupling } 4}+I_{\text {rotor }}
$$

where, $I_{\text {coupling2 }}, I_{\text {coupling3 }}, I_{\text {coupling4 }}$ are the rotary inertia of spindle when the second, third, fourth section of coupling rotate around spindle.

This simulation of this paper aims at low-velocity wind turbine, the main simulation parameters are showed below:

Table 1. Options for simulation parameters

\begin{tabular}{|c|c|}
\hline parameter & value \\
\hline Rated power/MW & 2 \\
\hline Rated wind speed $/ \mathrm{m} . \mathrm{s}-1$ & 9.5 \\
\hline Radius of impeller $/ \mathrm{m}$ & 55 \\
\hline Speed increasing ratio of gearbox & 91 \\
\hline Wind speed/m.s-1 & 12 \\
\hline Impeller rated rotate speed $/ \mathrm{rpm}$ & 13.3 \\
\hline
\end{tabular}

\section{Mass imbalance of impeller}

\subsection{Simulation of applying imbalance mass to different blade}

The model achieves its impeller imbalance through applying different mass on blade. We set five simulation working conditions and show it in Table 2 below to research on the influence caused by the direction distribution of impeller mass imbalance moment on wind turbine. The first working condition is the mass of three blades are totally equal, impeller is in the idea mass balance condition; and the second, third, fourth working condition correspond to put 200 kilogram mass on the same section of first, second, and third blade, respectively; the fifth working condition is corresponding to put $400 \mathrm{~kg}$ and $600 \mathrm{~kg}$ mass on the second and third blade respectively to form comparison with the four working condition above. The initial position of calculation blade for all the working condition correspond to three position of blade in Figure 1, the position of the first blade is zero position, with vertical direction. Wind wheel rotate clockwise, the $1,2,3,4,5$ here in this chapter correspond to working condition of $1,2,3,4,5$, respectively.

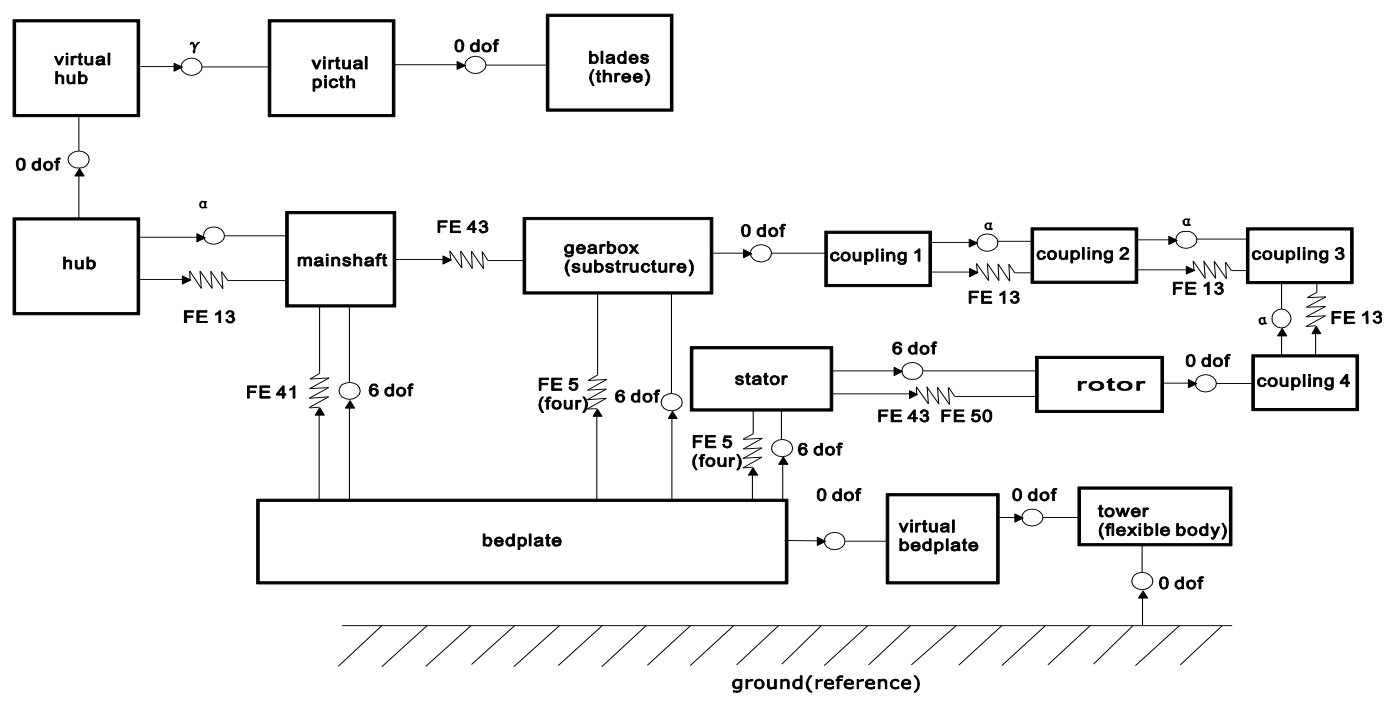

Figure 2. Structure picture of wind turbine topology. 
Under this five working conditions, in the time of simulation, the changing relationship of generator output power variation with time is showed in Figure 3, we can see that there exist larger differences of generator characteristic curve under different working condition. Figure 4 correspond to the characteristic curve when power of generator tends to be stable, working condition of 2,3,4,5 under imbalance condition, its value of power fluctuation range is obviously larger than the balanced state 1 , the same imbalance mass 2, 3, 4 have basically the same value of power fluctuation rang, the same as the change period, but exist some difference of phase, it's completely consistent with the analysis conclusion of the first chapter, so it also verify the correctness of multibody dynamic simulation model. The generating capacity of wind turbine generator during one period time is equal to the square surrounded by power curve and timer shaft, move forward from Figure 5, we can get during the period of starting wind turbine, the rank of generating capacity is $2 \approx 3>1>5>4$, we can know that not only the generating capacity is related to the size of imbalance mass, but also to its distribution position during the period of starting wind turbine. Because of the three blades which make up the impeller is unequal, as the impeller rotates, wind turbine will generate forced vibration due to suffering from alternating loads. Under the vibration, relatively incoming flow wind speed of impeller will have a period change to lead to wave of aerodynamic force. Wave of aerodynamic force will lead to the wave of impeller output torque, finally make influence on generator end electric power. The fluctuation range of electric power rank is $5>2 \approx 3 \approx 4>1$, we can get the size of fluctuation range is relevant with imbalance mass moment, as the imbalance mass moment turns bigger, the corresponding the electric power waves turn bigger. When apply equal imbalance mass moment to different blades, there is hardly change of electric power wave. When impeller is in equilibrium state, the corresponding electric power wave is very small. Period of wave average on $4.55 \mathrm{~s}$, and convert it into frequency is $0.22 \mathrm{~Hz}$. It is close to stable impeller rotate speed of $13.3 \mathrm{rpm}$ when the corresponding frequency is $0.222 \mathrm{~Hz}$, imbalance mass moment will lead to electric power wave when they have the same frequency. Wave frequency has nothing to do with the size and position of imbalance mass moment.

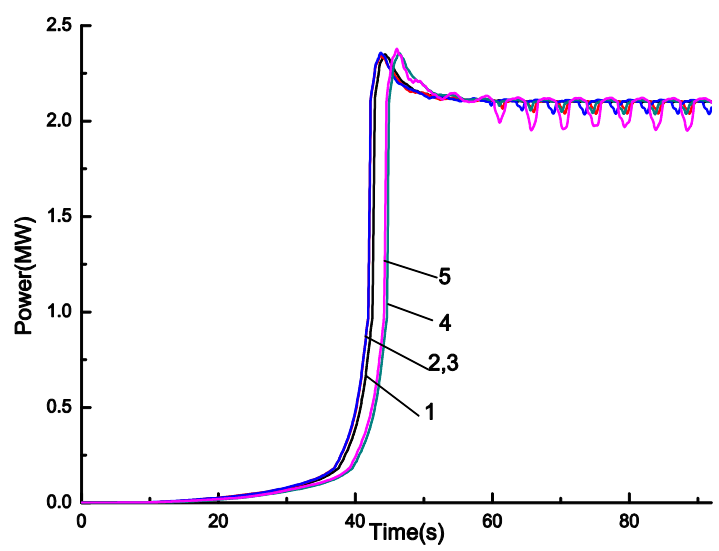

Figure 3. Power curve of generator.

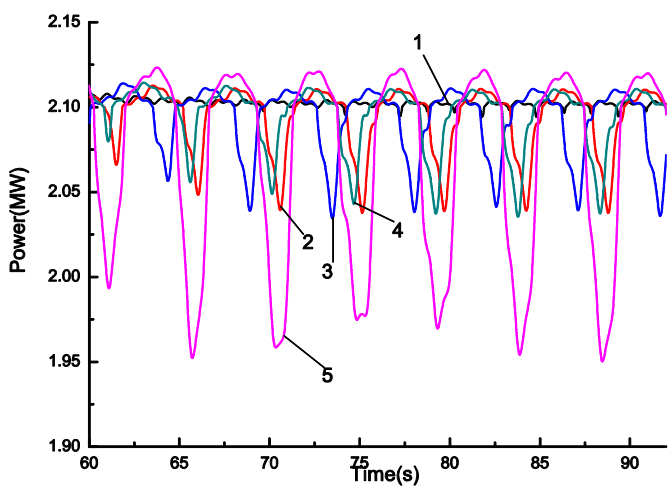

Figure 4. Electric power curve of stabled generator.

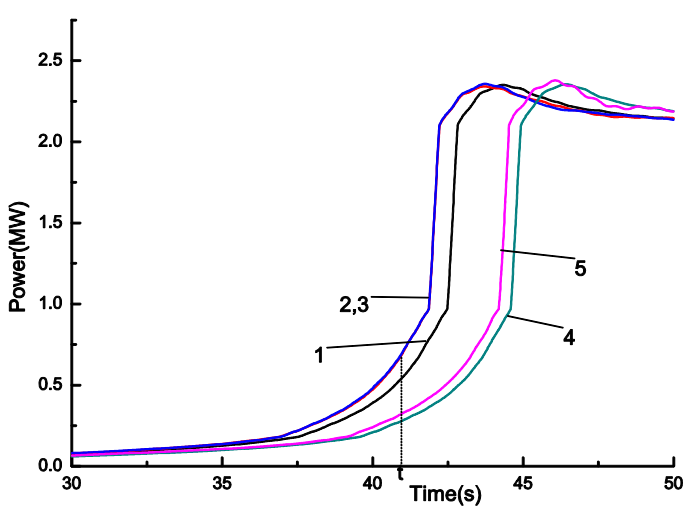

Figure 5. Generator power curve of the starting process.

\subsection{Apply different mass to the same blade}

Figure 6 represents the relevant curve between electric power fluctuation range of stabled generator and the size of imbalance mass moment in five working condition. We can see the amplitude of electric power wave has an approximate linear relation with imbalance mass moment. As the imbalance mass moment enlarge, the electric power fluctuation range will increase in proportion.

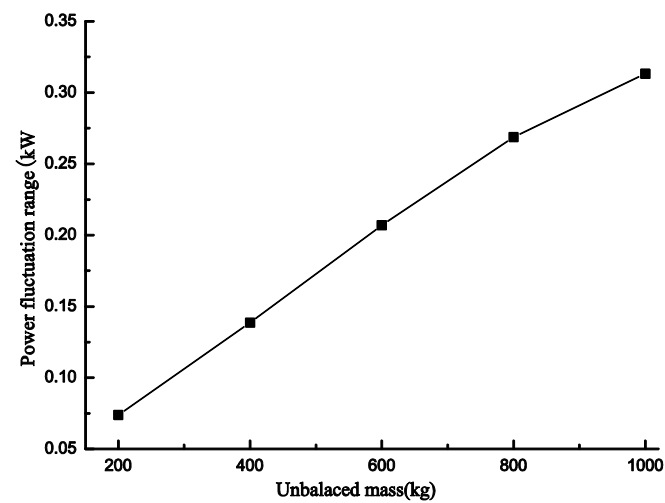

Figure 6. Relevant curve between electric power fluctuation range of generator and imbalance mass moment.

We set five simulation working condition in Table 3 below to research on the influences caused by imbalance mass moment of impeller on wind turbine generator, from working condition 1 to condition 5 correspond to apply $200 \mathrm{~kg}, 400 \mathrm{~kg}, 600 \mathrm{~kg}, 800 \mathrm{~kg}, 1000 \mathrm{~kg}$ to the same direction of the third blade respectively. The initial position of calculation blade for all the working condition 
correspond to three position of blade in Figure 1, the position of the first blade is zero position, with vertical direction. Wind wheel rotate clockwise, the 1,2,3,4,5 here in this chapter correspond to working condition of $1,2,3,4,5$,respectively.

The relationship of generator output power history under five different mass moments is showed in the Figure 7 below, we can see that there are differences in generator characteristic curve of different working conditions. Figure 8 represents the characteristic curve when generator tends to be stable, and as the imbalance mass moment enlarge, the electric power fluctuation range increase along. The generating capacity of wind turbine generator during one period time is equal to the square surrounded by power curve and timer shaft, move forward from Figure 7, we can get during the period of starting wind turbine, the rank of generating capacity is $1>2>3>4>5$, we can see that the generating capacity is related to the size of imbalance mass, the bigger imbalance mass moment is, the smaller generating capacity during starting process will be, it means mass imbalance of wind turbine makes direct influence on generating capacity during starting process. Period of wave average on $4.55 \mathrm{~s}$, and convert it into frequency is $0.22 \mathrm{~Hz}$. It is close to stable impeller rotate speed of $13.3 \mathrm{rpm}$ when the corresponding frequency is $0.222 \mathrm{~Hz}$, imbalance mass moment will lead to electric power wave when they have the same frequency. Wave frequency has nothing to do with the size of imbalance mass moment. In addition, there exist differences on phase position of electric power wave because of the imbalance mass will change the aerodynamic force to lead to phase deviation.

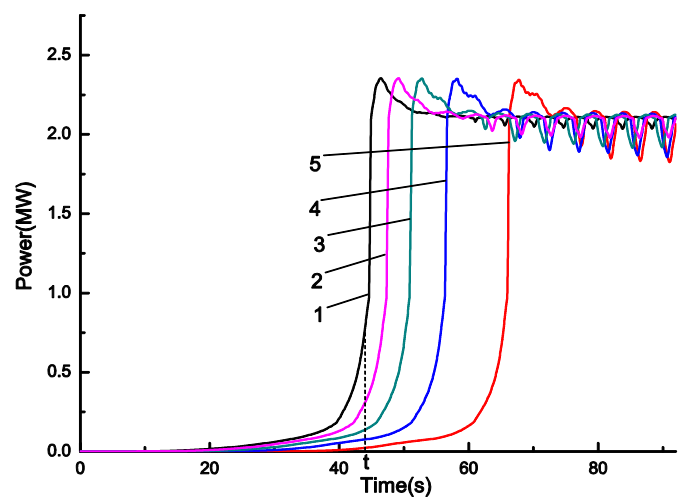

Figure 7. Electric power curve of generator.

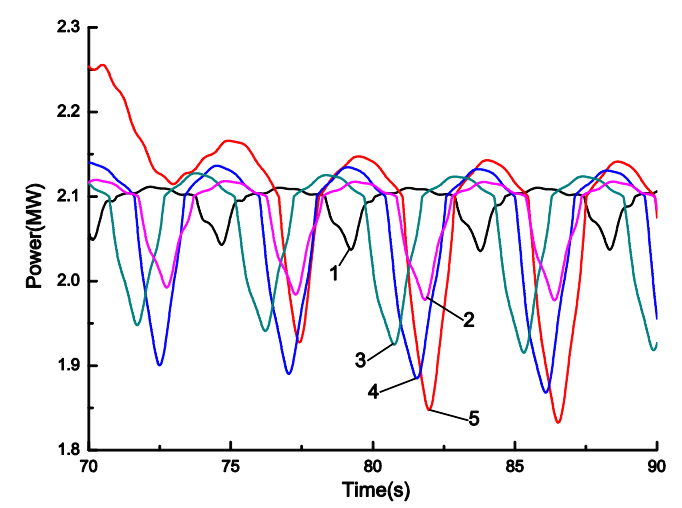

Figure 8. Electric power curve of stabled generator.

\section{Conclusions}

(1)This paper analyze calculation of wind turbine impeller mass moment in five different conditions, period of imbalanced moment inducted by different size of imbalance mass imposing blades at different position was the same as the wind turbine rotor, but the amplitude and phase was different, and we can infer backwards the mass imbalance situation according to size of amplitude and value of phase position. And we further infer the variation of output power is in proportion to imbalance mass moment of impeller, its change period is the same with the change period of imbalance mass moment

(2) This paper use software of SIMPACK to set up wind turbine generator virtual prototype model. The model includes impeller, hub, spindle, gearbox model and generator model, we can research on the influences of wind turbine generator caused by different sizes of imbalance mass on different blades through changing the size and position of imbalance mass. We verify the veracity of this model through testing that the simulation result of electric power variation period is the same as the inferred one.

(3) We analyze the generating capacity, rotate speed, variable pitch angle through model calculating of simulation. Not only generating capacity is related to the size of imbalance mass, but also to its distribution position during the period of starting wind turbine. Generator speed curve and variable pitch angle curve in stable condition have the same change rule with power curve. Both of them have the same wave frequency with impeller rotate frequency, the bigger imbalance mass moment is, the bigger fluctuation range will be.

\section{References}

1. Saidur R., Rahim N. A., Islam M. R., Solangi K.H. Environmental impact of wind energy[J]. Renewable and Sustainable Energy Reviews, 2011, 15(5): 2423 2430.

2. E Jia-qiang, LI Guang-ming, ZHANG Bin, DONG Jiang-Dong, ZHU Hao. Fatigue Simulation and Analysis on Planetary Gear of Megawatt Wind Power Yaw Reducer[J]. Advanced Materials Research, 2012, 455-456: 187-193.

3. Caselitz P, Giebhardt J. Rotor condition monitoring for improved operational safety of offshore wind energy converters $[\mathrm{J}]$. Journal of Solar Energy Engineering, 2005, 127(2): 253-261.

4. Ronny R, Jenny N. Imbalance estimation without test masses for wind turbines[J]. Journal of Solar Energy Engineering, 2009, 131(1): 1-7.

5. Jenny N, Ronny R, Thien $\mathrm{T}$ N. Mass and aerodynamic imbalance estimates of wind turbines[J].Energies, 2010, 3(4): 696-710.

6. Jiang Dongxiang. Theoretical and experimental study on wind wheel unbalance for a wind turbine[C]//2009 World Non-Grid-Connected Wind Power and Energy Conference, 24-26 Sept. 2009, Nanjing, China, 2009: 10975727.1-10975727.5. 
7. Zhao Minghao, Jiang Dongxiang, Li Shaohua. Research on fault mechanism of icing of wind turbine blades[C]//2009 World Non-Grid-Connected Wind Power and Energy Conference, 24-26 Sept. 2009, Nanjing , China, 2009: 10975712.110975712.4 .

8. Yang Tao, Ren Yong, Liu Xia, et al. Research on modeling and simulation of wind turbine rotor imbalance fault[J]. Journal of Mechanical Engineering, 2012, 48(6):130-135.
9. Ren Yong. Modeling and Simulation of the unbalance of wind turbine blade[D].Wuhan: Huazhong University of Science and Technology, 2012.

10. Guo Yuanchao, Xu Yiqing,Wang Fan, et al. Analysis of the influence of wind turbine mass unbalance on the load of wind turbine $[\mathrm{J}]$. Wind Energy, 2012(2): 70-72. 